\title{
Correction to: RF-EMF exposure induced by mobile phones operating in LTE small cells in two different urban cities
}

\author{
Taghrid Mazloum ${ }^{1}$ (D) $\cdot$ Sam Aerts $^{2} \cdot$ Wout Joseph $^{2} \cdot$ Joe Wiart $^{1}$
}

Published online: 19 December 2018

(C) Institut Mines-Télécom and Springer Nature Switzerland AG 2018

\section{Correction to: Annals of Telecommunications} https://doi.org/10.1007/s12243-018-0680-1

Table 2 was incorrectly captured in the original article, the correct Table 2 is shown below.

The original article has been corrected.

Table 2 Average values of RSRP and TX power (both in $\mathrm{mW}$ )

\begin{tabular}{|c|c|c|c|c|c|}
\hline & & \multicolumn{2}{|c|}{$\operatorname{RSRP}\left(\times 10^{-6} \mathrm{~mW}\right)$} & \multicolumn{2}{|c|}{ TX power $(\mathrm{mW})$} \\
\hline & & Annecy & Amsterdam & Annecy & Amsterdam \\
\hline \multicolumn{6}{|l|}{ AZQ } \\
\hline & $\mathrm{SC}$ & 0.020 & 0.398 & 13.18 & 3.31 \\
\hline & $\mathrm{MC}$ & 0.0006 & 0.039 & 40.74 & 41.88 \\
\hline \multicolumn{6}{|l|}{ JDSU } \\
\hline & $\mathrm{SC}$ & 0.006 & 0.251 & 28.12 & NA \\
\hline & $\mathrm{MC}$ & 0.0005 & 0.020 & 61.80 & 87.90 \\
\hline
\end{tabular}

The online version of the original article can be found at https://doi.org/ 10.1007/s12243-018-0680-1

Taghrid Mazloum

taghrid.mazloum@telecom-paristech.fr

1 Chair C2M, LTCI Télecom ParisTech, Université Paris Saclay, 46 rue Barrault, 75013 Paris, France

2 Ghent University/IMEC, Technologiepark-Zwijnaarde 15, 9052 Ghent, Belgium 